\title{
Modelo de Testagem Universal Aplicado à Área da Avaliação Psicológica
}

\author{
Cassandra Melo Oliveira ${ }^{1}$ \\ ${ }^{1}$ Faculdade CESUSC, SC, Brasil.
}

\author{
Carlos Henrique Sancineto da Silva Nunes ${ }^{2}$ \\ ${ }^{2}$ Universidade Federal de Santa Catarina, SC, Brasil.
}

\begin{abstract}
Resumo: O Desenho Universal (DU) é passível de ser adotado no planejamento de espaços, instrumentos e tecnologias que permitam o seu uso por pessoas com um escopo variado de características físicas e psicológicas. No presente artigo são descritos os desdobramentos alcançados pelo DU, ressaltando o desenvolvimento de instrumentos em Psicologia - Testagem Universal (TU). É delineado um roteiro para elaboração de testes segundo os princípios da TU. Uma das principais contribuições da Testagem Universal é a redução da necessidade de adaptações pós-elaboração do instrumental e consequente redução de custos com novos estudos. Ainda, alguns desdobramentos dos modelos de Desenho Universal são apresentados seguidos da perspectiva da diversidade cognitiva em Testagem Universal e a possibilidade de mediação destas demandas no processo de testagem. Finaliza com a proposta de um Modelo Integrativo de Testagem Universal.
\end{abstract}

Palavras-chave: Testagem Universal, Desenho Universal, Diversidade Cognitiva, Modelo Integrativo.

\section{Universal Testing Model Applied to the Area of Psychological Assessment}

\begin{abstract}
The Universal Design (DU) can be adopted in the planning of spaces, instruments and technologies that allow its use by people with a varied scope of physical and psychological characteristics. Inthe present article the DU achievements are described, emphasizing the development of instruments in Psychology - Universal Testing (TU). A road map for the preparation of tests according to TU principles is outlined. One of the main contributions of the Universal Test is the reduction of the need for post-instrument adaptation and consequent reduction of costs with new studies. Still, some developments of the Universal Design models are presented followed by the perspective of cognitive diversity in Universal Testing and the possibility of mediating the interference of these demands in the testing process. It ends with the proposal of an Integrative Model of Universal Testing.
\end{abstract}

Keywords: Universal Testing, Universal Design, Cognitive Diversity, Integrative Model.

\section{Modelo de Prueba Universal Aplicado al Área de Evaluación Psicológica}

Resumen: El Diseño Universal (DU) puede ser adoptado en la planificación de espacios, instrumentos y tecnologías que permitan su uso por personas con un variado alcance de características físicas y psicológicas. En el presente artículo se describen los logros de la DU, enfatizando el desarrollo de instrumentos en Psicología: evaluaciones diseñadas universalmente (EDU). Se describe una hoja de ruta para el desarrollo de pruebas de acuerdo con los principios de EDU. Una de las principales contribuciones de las Evaluaciones diseñadas universalmente es la reducción de la necesidad de adaptación posterior al instrumento y la consiguiente reducción de costos con nuevos estudios. Aún así, se presentan algunos desarrollos de los modelos de Diseño Universal seguidos por la perspectiva de la diversidad cognitiva en las Evaluaciones diseñadas universalmente y la posibilidad de mediación de estas demandas en el proceso de prueba. Termina con la propuesta de un Modelo Integrativo de Evaluaciones Diseñados Universalmente.

Palabras clave: Evaluación Universal, Diseño Universal, Diversidad Cognitiva, Modelo Integrativo. 


\section{Introdução}

O Desenho Universal surgiu no campo da arquitetura na busca de espaços que fossem projetados desde o início para todas as pessoas, incluindo indivíduos com ou sem deficiência, idosos e crianças. Mais recentemente (Shinn, \& Ofiesh, 2012; Thurlow, Lazarus, Christensen, \& Shyyan, 2016), foi incluído o pensar na diversidade cognitiva humana que abarcou indivíduos com TDAH (Transtorno de Déficit de Atenção e Hiperatividade), déficit cognitivo, dificuldades de aprendizagem, entre outros. Em sua expansão, o Desenho Universal alcançou produtos, projetos de espaços escolares até chegar ao ensino em si e concomitantemente aos processos avaliativos e seu instrumental. Assim, aporta-se na avaliação psicológica e nos testes psicológicos, foco principal deste artigo.

O conceito de Desenho Universal reflete a busca pela acessibilidade plena a espaços, produtos, materiais de ensino e ao currículo de ensino incluindo pessoas com e sem deficiência e tenta contemplar a diversidade corporal e cognitiva humana em um mesmo projeto/produto/material. A aplicação do Desenho Universal à avaliação escolar, à avaliação em larga escala e à avaliação psicológica apoiam-se em uma ampla literatura científica internacional (Albus, \& Thurlow, 2013; Almond et al., 2010; Burgstahler, 2001; Dolan, \& Hall, 2001; Dolan, Hall, Barnerjee, Chun, \& Stangman, 2005; Hanna, 2005; Johnstone, 2003; Ketterlin-Geller, 2005; Thompson, Johnstone, \& Thurlow, 2002; Thurlowet et al., 2016, entre outros). Os desdobramentos teóricos que serão abordados neste artigo contribuem para o reconhecimento e atendimento tanto da variação corporal quanto da diversidade cognitiva humana.

Tal conceito desdobrou-se em sete princípios os quais traduzem sua aplicação no campo da arquitetura e áreas afins (Burgstahler, 2001; Governo do Estado de São Paulo, 2010; Oliveira, Nuernberg, \& Nunes, 2013; Story, 2001): 1) o uso equitativo, tenta fornecer uso idêntico ou equivalente para uma ampla gama de usuários; 2) o uso flexível, possibilita a adaptabilidade, e acomoda uma ampla variedade de preferências, habilidades individuais e variações corporais; 3) o uso simples e intuitivo, que visa a eliminar a complexidade desnecessária e permitir a fácil compreensão e apreensão do espaço independente da experiência do usuário, de seu grau de conhecimento, habilidade de linguagem ou nível de concentração; 4) a informação de fácil percepção, que procura utilizar diferentes meios de comunicação, como informações sonoras e táteis; 5) a tolerância ao erro, a qual busca a segurança minimizando perigos de ações acidentais ou não intencionais; 6) o esforço físico mínimo, o qual procura dimensionar os produtos para que sejam utilizados de maneira confortável e com o mínimo de fadiga; e 7) o dimensionamento de espaços para acesso e uso abrangente, que busca o acesso e uso confortáveis para os usuários acomodando variações ergonômicas e oferecendo condições de manuseio diante de uma miríade de usuários.

Quando o Desenho Universal por meio do seu conceito e princípios é aplicado à Avaliação Psicológica e, em especial, aos testes psicológicos, obtém-se a Testagem Universal (Universal Assessment) (Thompson et al., 2002). Assim, na busca pela acessibilidade plena ou máxima possível aos processos avaliativos, os sete princípios do Desenho Universal foram transformados em sete princípios da Testagem Universal. Ressalta-se, entretanto, que não há uma correspondência direta e exclusiva, isto é, em um princípio da Testagem Universal pode ser contemplado um ou mais princípios do Desenho Universal. Os sete princípios da Testagem Universal em sua definição constitutiva são (Johnstone, 2003; Oliveira et al., 2013; Thompson et al., 2002):

1) População de avaliação ampla e inclusiva

Os testes psicológicos são desenvolvidos ou adaptados objetivando uma população ampla, geralmente pessoas com e sem deficiências -incluindo, por exemplo, a situação em que o psicólogo é uma pessoa com deficiência e pretende usar o teste para avaliar outra pessoa (com deficiência ou não). Atende a uma demanda crescente por maior inclusão da diversidade humana. Princípios do Desenho Universal refletidos: Uso equitativo e Uso flexível.

2) Definição precisa do construto

Os testes psicológicos são desenvolvidos ou adaptados visando a máxima precisão na definição dos construtos a serem medidos, evitando que possam ser afetados por outros construtos alheios ao que se pretende medir. Para tanto, faz-se imprescindível que os instrumentos empreguem teorias fortemente embasadas. Evita-se, assim, a construção de barreiras proporcionadas por conteúdo irrelevante semelhantemente às barreiras físicas que são evitadas nos projetos do Desenho Universal aplicados à arquitetura. Por exemplo, em um teste que envolva originalmente a apresentação de figuras geométricas aos testandos, 
será que a apresentação dessas figuras em relevo para pessoas cegas avaliaria a mesma habilidade? Considera-se, neste caso, que a percepção por meio do tato pode ser muito diferente do estímulo para o qual o teste foi originalmente desenvolvido - acarretando a interferência de um novo construto. Os testes de Testagem Universal procuram desde o seu desenvolvimento evitar que tais aspectos indesejados ocorram. Princípios do Desenho Universal refletidos: Uso equitativo e Tolerância ao erro.

3) Itens acessíveis e não tendenciosos

Os testes psicológicos são desenvolvidos ou adaptados para que indivíduos pertencentes a diferentes grupos (deficientes, estrangeiros, sem deficiência, entre outros) com a mesma habilidade no construto avaliado tenham a mesma chance de acertar os itens do teste psicológico (em testes de desempenho) ou a apresentarem certos padrões de respostas (em testes de personalidade, interesse, entre outros), e que todos estes grupos compreendam as instruções dos procedimentos envolvidos na testagem. A construção dos itens e as instruções procuram evitar o viés cultural e alcançar a magnitude perceptiva do usuário. Princípios do Desenho Universal refletidos: Uso equitativo e Uso flexível.

4) Testes flexíveis a acomodações

Em alguns casos, mesmo que o teste tenha sido construído segundo os princípios da Testagem Universal, é necessária a adoção de adaptações extras para viabilizar o uso do mesmo com pessoas com características físicas ou psicológicas específicas. A vantagem do teste que foi construído segundo a Testagem Universal é permitir o uso destas acomodações de modo que admita a comparabilidade dos resultados entre as aplicações com e sem adaptações extras. Por exemplo, um instrumento que seja apresentado no computador e possam ser agregados recursos de áudio, informações extras por meio de acesso a material adicional e acréscimo no tempo - sendo, todas estas possibilidades pertencentes ao projeto do teste e a seus estudos de desenvolvimento e subsequentes. Tal perspectiva parte da possibilidade de um formato flexível que agregue novos estudos ao longo do tempo. Princípios do Desenho Universal refletidos: Uso equitativo, Uso flexível, Informação de fácil percepção e Dimensionamento de espaços para acesso e uso abrangente.

5) Instruções e procedimentos simples, claros e intuitivos

Os testes psicológicos são desenvolvidos ou adaptados evitando-se que instruções e procedimentos em linguagem complexa ou confusa prejudiquem a compreensão do que é solicitado, evitando que o testando incorra em erros por não compreender o que lhe é pedido. Princípios do Desenho Universal refletidos: Uso equitativo, Uso simples e intuitivo, Informação de fácil percepção e Tolerância ao erro.

6) Leitura agradável e de máxima inteligibilidade

Os testes psicológicos são desenvolvidos ou adaptados para reduzir a complexidade verbal e de organização textual dos itens e instruções preservando seu conteúdo essencial. Princípios do Desenho Universal refletidos: Uso equitativo, Uso flexível e Uso simples e intuitivo.

7) Máxima legibilidade

Os testes psicológicos são desenvolvidos ou adaptados de forma que a aparência física do texto, os gráficos, tabelas e ilustrações, bem como o formato das respostas possam ser percebidos e decifrados com facilidade. Princípios do Desenho Universal refletidos: Uso equitativo, Uso simples e intuitivo, Informação de fácil percepção, Esforço físico mínimo e Dimensionamento de espaços para acesso e uso abrangente.

A Testagem Universal preocupa-se com o formato dos testes e estuda a sua influência na realização do mesmo e foca as adaptações como proporcionadoras de acessibilidade, analisando sua repercussão na qualidade dos instrumentos. Uma diferença básica entre Testagem Psicológica e Testagem Universal é que a Testagem Psicológica pretende tornar o teste claro e conciso para grupos populacionais específicos, já a Testagem Universal busca um design, um formato de teste, que possa acomodar-se a uma população ampla com o máximo de acessibilidade possível.

\section{Método}

Foram realizadas pesquisas em bases de dados nacionais e internacionais, sobretudo nas segundas, uma vez que a literatura nacional é escassa nesta temática. A verificação das publicações conforme o ano, 1998 até 2016, utilizou, principalmente, as seguintes palavras-chave: acessibility, universal design, universal assessment, cognitive diversity $\mathrm{e}$ disability. Os textos que compõem o presente trabalho foram selecionados conforme a proximidade com a temática avaliação/testagem e Desenho Universal, abrangendo as relações e desdobramentos da interlocução entre ambas. 


\section{Resultados e discussões}

\section{A construção e adaptação de instrumentos de Testagem Universal}

Quando a Testagem Universal é aplicada ao desenvolvimento de instrumentos de avaliação desde o início de seu projeto, há a redução da necessidade de adaptações pós-elaboração e, consequente, redução de tempo e custos com novos estudos. Além disso, possibilita-se, o que é mais importante, uma avaliação de qualidade para uma população ampla incluindo pessoas com deficiência (Dolan et al., 2005; Johnstone, 2003; Ketterlin-Geller, 2005; Thompson et al., 2002). Contudo, como aplicar os princípios da Testagem Universal com vistas a alcançar um maior número de recursos auxiliares e potencializadores da capacidade de proporcionar acessibilidade?

O uso do computador pode ser um grande aliado para os desenvolvedores de testes que procuram aplicar os princípios da Testagem Universal na construção de instrumentos mais acessíveis, uma vez que permite criar um ambiente que proporciona maiores possibilidades de variação, isto é, maior flexibilidade no uso, escolha de formatos, tamanhos de fontes, além do fato de que admite o acoplamento de tecnologias assistivas que venham a complementar e potencializar a Testagem Universal. Porém, a Testagem Universal pode também ser aplicada satisfatoriamente ao formato tradicional - lápis e papel (Ketterlin-Geller, 2005; Dolan, \& Hall, 2001). O ponto chave está nas possibilidades viabilizadas pelo computador que são superiores às do formato lápis e papel. Ressalta-se, entretanto, que se o testando não estiver familiarizado com o computador este poderá se transformar em barreira em vez de um recurso vantajoso.

No State Guide to the Development of Universally Designed Assessments (Johnstone, Altman, \& Thurlow, 2006) é descrito um roteiro para elaboração de testes segundo os princípios da Testagem Universal, o qual será exposto de forma resumida nos parágrafos subsequentes com algumas contribuições do estudo de Oliveira (2013). Assim sendo as etapas envolvidas na elaboração de instrumentos de Testagem Universal são (Johnstone et al., 2006; Oliveira, 2013):

1) A conceituação do teste: com a escolha do construto a ser medido e a sua definição explícita e precisa. Alguns questionamentos norteadores para construção de instrumentos de Testagem Universal: Este construto é realmente relevante? Aplica-se para esse público? Possui literatura que o embase?
2) Escolha do número e tipo de itens, minimização do efeito de fatores externos ao construto: no que se refere ao formato, cada item deve ser desenvolvido de forma a ser compatível com recursos de acessibilidade. Já no tocante ao conteúdo, deve-se evitar qualquer aspecto que possa oferecer vantagem ou desvantagem para qualquer subgrupo da amostra ao qual se destina o teste. Isto implica em possuir na equipe de desenvolvimento do instrumento pessoas especializadas na construção de itens para grupos variados. A fim de minimizar o efeito de fatores externos (por exemplo: evitar o uso de gráficos ou figuras que não sejam transponíveis para o Braille, ou evitar complexidade linguística desnecessária). Alguns questionamentos norteadores: O conteúdo de algum item oferece vantagem ou desvantagem a um grupo específico? Os formatos dos itens são acessíveis ou possuem recursos proporcionadores de acessibilidade?

3) Análise e correções: consiste em submeter o instrumento a uma equipe de revisão de testes. A equipe deve possuir indivíduos peritos com características semelhantes à população a que se destina o teste, pessoas com as diferentes deficiências alvo, os quais sejam pesquisadores e profissionais especialistas no construto a ser medido. Nesta etapa é realizada a revisão de conteúdo, formato e design do instrumento, e, deste modo, a análise e correção de possíveis falhas até o momento (por exemplo: verificação do funcionamento de software leitores de tela ou outro recurso de leitura do instrumento, como voz gravada ou vídeo). Além da revisão pela equipe de pesquisa, é importante a avaliação do instrumento por uma equipe de juízes externos, o que implica no uso da metodologia da Análise de Juízes (Urbina, 2007). Alguns questionamentos norteadores: Os recursos de acessibilidade estão funcionando corretamente? O formato está adequado? O conteúdo se coaduna com o objetivo desejado? $\mathrm{O}$ design do instrumento está em consonância com os princípios da Testagem Universal? No link que segue, disponibiliza-se um checklist dos principais elementos da Testagem Universal com vistas a auxiliar o processo de revisão do teste desenvolvido pelos autores deste artigo (http://lpap.paginas.ufsc.br/?page_id=382 ).

4) A aplicação em campo: pode assumir vários modelos, como por exemplo, ser realizada amostras pessoas com ou sem deficiência ou para grupos específicos. A escolha do modelo de estudo de campo ou estudo-piloto e, consequentemente, da amostra dependerá dos objetivos do teste, do construto medido e da ampli- 
tude do público-alvo, como por exemplo, pessoas com deficiências variadas e pessoas sem deficiência - ambas de vários grupos populacionais. Perguntas norteadoras: Como será o estudo-piloto? Qual a amplitude da amostra?

5) Análises qualitativas e quantitativas: as análises qualitativas estão voltadas para a acessibilidade e usabilidade do instrumento e como estas são percebidas por seus usuários. Já as análises quantitativas permeiam a utilização de recursos estatísticos variados incluindo estatísticas descritivas e multivariadas e, ainda, a Teoria de Resposta ao Item (TRI), a qual é reconhecida como uma técnica de análise estatística valiosa nos estudos da aplicação do Desenho Universal a instrumentos de avaliação, sendo também possível utilizar-se de técnicas da Teoria Clássica dos Testes (TCT). De forma mais específica, ressalta-se o uso do Funcionamento Diferencial do Item (DIF) para verificar a equivalência do funcionamento dos itens nos grupos e formatos adotados e a análise fatorial confirmatória para verificar a manutenção da estrutura fatorial (Embretson, \& Reise, 2000). Perguntas Norteadoras: Quais análises qualitativas serão úteis para este instrumento? Quais análises quantitativas se aplicam a esse instrumento?

6) Revisão final: são realizados os últimos ajustes do instrumento e produz-se a sua versão final. Perguntas norteadoras: A versão final alcançou o objetivo desejado? Será necessário retornar a alguma etapa anterior para alcançar a versão final?

\section{Aplicação da Testagem Universal no Brasil}

Oliveira (2013), seguindo a perspectiva da Testagem Universal, realizou o estudo da adaptação de um teste psicológico para avaliação da personalidade informatizado à Testagem Universal. O teste de personalidade informatizado adaptado procurou atender aos sete princípios do Desenho Universal/Testagem Universal. Tal instrumento foi submetido a uma amostra de 146 indivíduos com deficiência visual e 150 indivíduos sem deficiência.

A Bateria Informatizada para Avaliação da Personalidade, instrumento que teve o seu formato adaptado aos princípios do Desenho Universal/Testagem Universal, baseia-se no modelo dos cinco grandes fatores da personalidade (Extroversão, Socialização, Neuroticismo, Realização e Abertura). Seus itens são de autorrelato e fazem parte do banco de itens do Labora- tório de Pesquisa em Avaliação Psicológica (LPAP) da Universidade Federal de Santa Catarina (UFSC).

Complementarmente foi desenvolvido um instrumento para avaliação da aplicação do Desenho Universal primando por avaliar os princípios do Desenho Universal/Testagem Universal. As adaptações foram realizadas na estrutura do formato de aplicação do teste: 1 . Orientações dadas para execução do teste; 2. Formato dos itens; 3 . Tamanho da fonte e tipo de letra; 4. Cores e espaços; e 5. Ordem de apresentação - objetivando atender ao público com deficiência visual. Apesar das adaptações priorizarem o atendimento ao público com deficiência visual, foi objetivo do estudo desenvolver um formato do teste para o Desenho Universal/Testagem Universal e, desta forma, os recursos foram desenvolvidos para atender também às pessoas sem deficiência.

As pessoas com deficiência visual frequentemente empregam os leitores de tela quando da utilização do computador e da Internet. Os leitores de tela são proporcionadores de acessibilidade transformando texto em voz sintetizada e apresentando recursos para navegação. A forma adaptada e o instrumento para avaliação do Desenho Universal/Testagem Universal foram testados pelos pesquisadores com cinco tipos de leitores de tela/sintetizadores de voz com o objetivo de verificar se os itens se adequavam a estes recursos de Tecnologia Assistiva. Durante este processo, palavras e formatos que não eram flexíveis aos leitores foram modificados.

Os instrumentos e seus vários formatos possíveis (itens, cores e fontes) foram submetidos primeiramente à equipe de pesquisa composta por dois pesquisadores sem deficiência visual e dois especialistas cegos, sendo uma psicóloga e um professor de informática para deficientes visuais. Formatos variados de itens foram testados e, posteriormente, foi definida uma forma adaptada para o estudo-piloto.

Novas correções foram realizadas sendo obtida a forma final do instrumento, o qual foi aplicado na amostra de pesquisa. Ressalta-se ainda, que a escolha nesta pesquisa pelo modo de apresentação do teste adaptado como uma ferramenta informatizada online perpassou por vários princípios do Desenho Universal, pois permitiu o acesso ao teste por pessoas com e sem deficiência utilizando seu computador pessoal independentemente da distância que os indivíduos estavam do local de origem da pesquisa (UFSC) viabilizando um uso flexível e equitativo. 
Os resultados do instrumento que avaliou a adaptação do instrumento para adaptação da Testagem Universal apontaram para o atendimento de todos os sete princípios empregados e concomitantemente a acessibilidade do instrumento para indivíduos com e sem deficiência visual. Alcançou-se a acessibilidade máxima possível para uma população ampla e inclusiva. No entanto, o teste adaptado à Testagem Universal possuiu como limitação o fato de não se aplicar a outros tipos de deficiência, o qual, para tanto, necessitará de novos estudos.

Vários autores (Amiralian, 1997; Case, Zucker, \& Jeffries, 2005; Kastrup, Sampaio, Almeida, \& Carijó, 2009) apontam para a ineficiência das adaptações realizadas em testes psicológicos para os indivíduos com deficiência visual uma vez que os mesmos geralmente partem da perspectiva dos videntes. A pesquisa de Oliveira (2013) relatada buscou partir da perspectiva do deficiente visual, de sua forma de utilização do computador, dos leitores de tela e como o uso destas tecnologias repercutem nos formatos dos itens e na forma de apresentação das instruções. Considera-se que tal ponto de partida contribuiu para o alcance dos resultados positivos reportados, ou seja, a acessibilidade segundo os princípios da Testagem Universal.

\section{Neurocognição e avaliações de Testagem Universal}

A diversidade cognitiva segundo Shinn e Ofiesh (2012) consiste nos diferentes traços cognitivos (por exemplo, memória, atenção e velocidade de processamento) de cada indivíduo. Os referidos autores preocuparam-se com a variabilidade cognitiva no contexto da aprendizagem assumindo que tal complexidade cognitiva inclui pessoas com e sem deficiências e ainda indivíduos com dislexia, TDAH, déficits cognitivos etc. Observa-se que a ideia de uma diversidade cognitiva possui ampla consonância com a perspectiva do Desenho Universal uma vez que toca à diversidade humana e procura incluí-la em vários contextos, sobretudo o educacional. A partir deste último, alcança os processos avaliativos e seus instrumentais.

Shinn e Ofiesh (2012) dividem as demandas cognitivas relacionadas ao processo de testagem em duas categorias: 1) Acessar o teste - o conteúdo e formato (test access): a compreensão da linguagem, o raciocínio visuoespacial e a fluência no conteúdo do teste são as características cognitivas mais exigidas; 2) $\mathrm{O}$ processo de responder ao teste (test output): Integra- ção visuo-motora e a memória de longo prazo estariam mais implicadas nesse processo. Assim, observa-se que tal categorização expressa às áreas que estão primariamente relacionadas aos processos cognitivos. Contudo, tanto no acessar como no responder serão necessárias a interações de todas estas demandas cognitivas.

As demandas cognitivas parecem variar em um espectro no qual está presente tanto o processo de acessar o teste como de respondê-lo. O desempenho do indivíduo será baixo caso o indivíduo consiga acessar o teste, mas não consiga respondê-lo e também se ele não conseguir nem mesmo acessar o instrumento. $\mathrm{O}$ acréscimo das demandas cognitivas à execução de instrumentos decorre, entre outras variáveis, do aumento da dificuldade do teste. Quanto menos acessíveis forem os testes, mais eles irão demandar cognitivamente de modo indesejado. Assim, testes acessíveis possibilitam que indivíduos com os mais variados níveis de proficiência no construto medido o realizem, medindo realmente o que se pretende medir sem a interferência de variáveis estranhas; este o intuito da aplicação do Desenho Universal aos instrumentos avaliativos, dentre eles, os testes psicológicos.

\section{Modelo integrativo de Testagem Universal}

Objetivando sintetizar as principais ideias que permeiam este artigo com vistas a alcançar um modelo integrativo de Testagem Universal, faz-se imprescindível o conhecimento das variáveis que compõem o processo de testagem. Assim, foram selecionadas algumas das variáveis para que o testando acesse e responda a um instrumento agrupando-as nas seguintes categorias (Vide Figura 1).

1) Demandas Cognitivas: envolvem todos os aspectos referentes às demandas da memória de trabalho, atenção, velocidade de processamento etc. Tais demandas causam interferência no processo de testagem por serem ao mesmo tempo mediadoras (o processamento da informação depende de cada uma delas) e executoras (a realização da tarefa também é dependente de cada uma delas, bem como o resultado final) na execução de instrumentos avaliativos.

2) Habilidades motoras: possibilitam a expressão da cognição em sua amplitude. Indivíduos com deficiência que afetam suas habilidades motoras, mesmo possuindo alto nível de proficiência no construto medido, ficam impossibilitados de demonstrar suas 
habilidades. Por exemplo, um grande cientista com deficiência, como Stephen Hawking, sem acessibilidade ficaria impossibilitado de demonstrar suas reais habilidades em um teste de aritmética simples, no formato lápis e papel.

3) Órgãos dos sentidos e percepções: os órgãos dos sentidos têm um papel crucial na percepção do que é apresentado. Caso o teste não vislumbre as diferentes possibilidades de percepção do material (por exemplo, visual, auditiva, táctil, em Braille e em língua de sinais), ele não será acessível para a diversidade cognitiva humana.

4) Aprendizagem: as formas como os indivíduos aprendem irão influenciar o modo como eles respondem aos testes, por exemplo: aprendizagem guiada por imagens, aprendizagem guiada por textos, percepção das partes primeiro ou percepção do todo primeiro, etc. Portanto, o formato do instrumento faz-se importante neste tocante, espera-se que instrumentos que considerem diferentes tipos de aprendizes tenham maior potencial de acessibilidade. Ademais, o fato de já ter passado por tarefas semelhantes irá influenciar os seus resultados em testes futuros, ou seja, em atividades similares.

5) Ambiente: um meio estimulante, desencadeante de novos desafios, aumenta o sentimento de autoeficácia dos indivíduos sejam eles com ou sem deficiência. Assim, o estímulo à realização de atividades variadas e das próprias atividades da vida diária (AVDs) podem contribuir para o engajamento dos indivíduos em atividades futuras. Para tais atividades, sejam novas ou repetições de tarefas já realizadas, espera-se que um ambiente estimulante de testagem favoreça a realização destes instrumentais. Ainda, experiências em meios acessíveis podem estimular o sentimento de realização do indivíduo, mudando o paradigma de compreensão do espaço e de si mesmo, ou seja, a inacessibilidade está no meio externo e não no indivíduo por tratar-se de pessoa com deficiência ou com uma demanda cognitiva específica.

6) Meio Familiar: que promove a independência e o protagonismo e possui qualidades que o aproximam do desenho universal. Assim, o meio familiar favorece e media processos promotores de acessibilidade por encararem o indivíduo como capaz impedindo a estagnação. Espera-se que o meio familiar seja um promotor de acessibilidade por excelência uma vez que é nele que o indivíduo se desenvolve. Como tal, o engajamento do indivíduo e sua familiaridade com recursos de acessibilidade irá favorecer a experiência e o engajamento na busca do Desenho Universal e concomitantemente da Testagem Universal.

7) Tecnologias Assistivas: responsáveis pela mediação do processo de acessibilidade. Coadunam-se com a acessibilidade plena proposta pelo Desenho Universal/Testagem Universal uma vez que, quando inteligentemente utilizadas, permitem que os indivíduos realizem as mais diversas atividades sem a necessidade de auxílio externo, isto é, independentemente. São recursos potentes na elaboração de projetos de DU/TU tanto no que se refere a espaços e produtos quanto aos testes psicológicos.

As categorias descritas contribuem para a compreensão do modelo de Testagem Universal, o qual não depende de duas variáveis apenas, quais sejam: o teste e o indivíduo, de forma alguma. $\mathrm{O}$ processo de testagem é bem mais complexo e a criação de ferramentas que primam pela qualidade para grupos amplos requer o desdobrar de um olhar ampliado para o fenômeno da testagem e da avaliação psicológica.

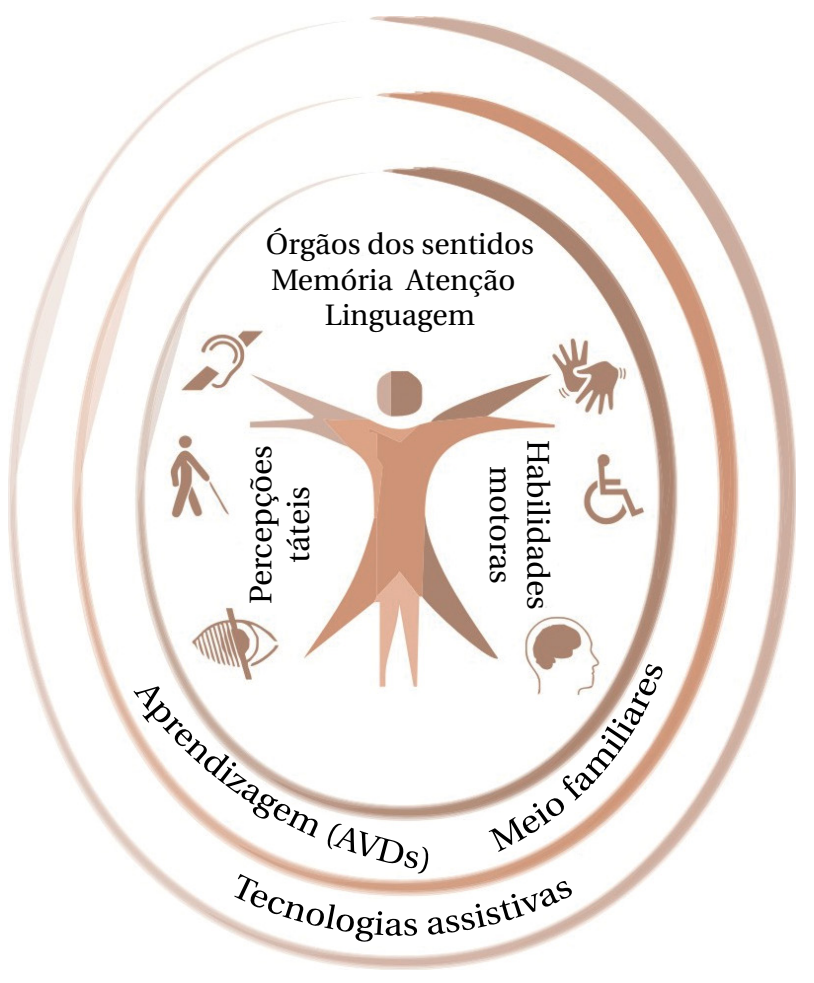

Figura 1

Categorias do Modelo Integrativo de Testagem Universal. 


\section{Conclusões}

O presente artigo detalhou o surpreendente alcance dos princípios do Desenho Universal, inicialmente elaborados com o foco em projetos de arquitetura. Os mesmos evoluíram teoricamente e mostraram a utilidade de sua aplicação em outras áreas. Na Educação, compreendeu um campo vasto com aplicações que vão desde o espaço físico até o currículo e o processo de aprendizagem. As aplicações do Desenho Universal aos testes psicológicos e ao processo de testagem foram os enfoques principais deste trabalho, os quais se tratam de um campo amplo de possibilidades, criador de uma forma diferenciada de vislumbrar a testagem para além do momento ou fração temporal em que ele ocorre, visando, assim, abarcar as inúmeras variáveis que podem interferir neste processo. Faz-se, para tanto, de fundamental importância uma metodologia de construção de testes de Testagem Universal que permitam a segurança técnica necessária a esta tarefa.

Conforme apresentado, as etapas envolvidas na construção de testes de Testagem Universal são semelhantes às etapas já estandardizadas na construção de instrumentos psicológicos. Acresce-se que o conteúdo de cada etapa ou suas subetapas assumem maior riqueza e detalhamento de aspectos estruturais, sobretudo em relação ao formato e a sua adequação à utilização de tecnologias assistivas e a populações amplas. Em se tratando da Testagem Universal, torna-se uma preocupação permanente a acessibilidade do instrumento tanto em relação ao formato quanto ao seu conteúdo, bem como a operacionalização dos princípios da Testagem Universal em cada etapa do processo de construção. Consequentemente, a Testagem Universal permite a ampliação da qualidade dos instrumentos para grupos amplos.

A Testagem Universal ultrapassou a visão do testando estático e em seus desdobramentos mais atuais considerou as demandas cognitivas dos indivíduos. Tais demandas possibilitaram a ampliação do grau de detalhamento do processo de realização de testes, dividindo-o no processo de acessar e de responder ao teste, uma vez que apesar destes dois processos possuírem algumas demandas cognitivas que se assemelham, possuem outras demandas bastante diversas. Logo, o pensar em uma população ampla e inclusiva de Testagem Universal ultrapassa o pensar apenas em nível de pessoas com e sem deficiência, mas abarca a diversidade cognitiva humana e reforça o compromisso ético dos profissionais de Psicologia com a qualidade destes materiais. Espera-se, por fim, que este trabalho contribua para ampliar o olhar em relação à elaboração de instrumentos em Psicologia e que a Testagem Universal alcance não só os instrumentos, mas as atitudes e o pensamento dos profissionais de Psicologia, os quais incluam estudos desta natureza quando do processo de desenvolvimento de seus instrumentais.

\section{Referências}

Albus, D., \& Thurlow, M. L., (2013). Accommodation policies for states' alternate assessments based on alternate achievement standards (AA-AAS) (Synthesis Report 90). Minneapolis, MN: University of Minnesota, National Center on Educational Outcomes.

Almond, P., Winter, P., Cameto, R., Russell, M., Sato, E., Clarke-Midura, J. et al. (2010). Technology-enabled and universally designed assessment: Considering access in measuring the achievement of students with disabilities: A foundation for research. Journal of Technology, Learning, and Assessment, 10(5). Recuperado de https://ejournals.bc.edu/ojs/index.php/jtla/article/view/1605/1453

Amiralian, M. L. T. M. (1997). Compreendendo o cego: Uma visão psicanalítica da cegueira por meio de desenhos-estórias. São Paulo, SP: Casa do Psicólogo.

Burgstahler, S. (2001). Equal access: Universal design of instruction: A checklist for inclusive teaching. Washigton, DC: University of Washington. Recuperado de http://www.washington.edu/doit/Brochures/Academics/equal_ access_udi.html

Case, B. J., Zucker, S., \& Jeffries, J. L. (2005). A primer on assessing the visually impaired. San Antonio , TX: Harcourt Assessment. 
Dolan, R. P., \& Hall, T. E. (2001). Universal design for learning: Implications for large-scale assessment. IDA Perspectives, 27(4): 22-25.

Dolan, R. P., Hall, T. E., Banerjee, M., Chun, E., \& Strangman, N. (2005). Applying principles of universal design to test delivery: The effect of computer-based read-aloud on test performance of high school students with learning disabilities. Journal of Technology, Learning, and Assessment, 3(7). Recuperado de https://ejournals.bc.edu/ojs/ index.php/jtla/article/view/1660/1496

Embrestson, S. E., \& Reise, S. P. (2000). Item response theory for psychologists. New Jersey, NJ: Lawrence Erlbaum.

Governo do Estado de São Paulo, Secretaria de Estado da Habitação \& Secretaria de Estado dos Direitos da Pessoa com Deficiência (2010). Desenho universal: habitação de interesse social. São Paulo, SP: Secretaria de Estado da Habitação.

Hanna, E. L. (2005). Inclusive design for maximum accessibility: A practical approach to universal design. Pearson Educational Measurement. Recuperado de http://images.pearsonassessments.com/images/tmrs/tmrs_rg/ RR_05_04.pdf

Johnstone, C. J. (2003). Improving validity of large-scale tests: Universal design and student performance (Technical Report 37). Minneapolis, MN: University of Minnesota.

Johnstone, C., Altman, J. \& Thurlow, M. (2006). A state guide to the development of universally designed assessments. Minneapolis, MN: University of Minnesota.

Kastrup, V., Sampaio, E., Almeida, M. C., \& Carijó, F.H. (2009). O aprendizado da utilização da substituição sensorial visuo-tátil por pessoas com deficiência visual: primeiras experiências e estratégias metodológicas. Psicologia \& Sociedade, 21(2): 256-265. https://doi.org/10.1590/S0102-71822009000200013.

Ketterlin-Geller, L. R. (2005). Knowing what all students know: Procedures for developing universal design for assessment. Journal of Technology, Learning and Assessment, 4(2). Recuperado de https://ejournals.bc.edu/ojs/ index.php/jtla/article/view/1649

Oliveira, C. M. (2013). Evidências de validade de uma bateria informatizada para avaliação da personalidade adaptada ao desenho universal (dissertação). Universidade Federal de Santa Catarina, Florianópolis, SC.

Oliveira, C. M., Nuernberg, A. H., \& Nunes, C. H. S. S. (2013). Desenho universal e avaliação psicológica na perspectiva dos direitos humanos. Avaliação Psicológica, 12(3), 421-428.

Shinn, E., \& Ofiesh, N. S. (2012). Cognitive diversity and the design of classroom tests for all learners. Journal of Postsecondary Education \& Disability, 25(3), 227-245.

Story, M. F. (2001). Principles of universal design. In: W. F. E. Preiser, \& E. Ostroff, Universal design handbook (pp. 158-220). New York, NY: McGraw-Hill Professional.

Thompson, S. J., Johnstone, C. J., \& Thurlow, M. L. (2002). Universal design applied to large scale assessments (Synthesis Report 44). Minneapolis, MN: University of Minnesota.

Thurlow, M. L., Lazarus, S. S., Christensen, L. L., \& Shyyan, V. (2016). Principles and characteristics of inclusive assessment systems in a changing assessment landscape (NCEO Report 400). Minneapolis, MN: University of Minnesota,.

Urbina, S. (2007). Fundamentos de testagem psicológica. Porto Alegre, RS: Artes Médicas.

\section{Cassandra Melo Oliveira}

Docente do curso de Psicologia da Faculdade CESUSC, Florianópolis -SC. Brasil.

E-mail: casymelo@gmail.com

\section{Carlos Henrique Sancineto da Silva Nunes}

Docente do Departamento de Psicologia da Universidade Federal de Santa Catarina (UFSC), Florianópolis -SC.

Brasil.

E-mail: carlos.nunes@ufsc.com.br 
Endereço para envio de correspondência:

Rua Aroeira da Praia, 45, apto. 104. Rio Tavares. CEP: 88048-644.

Florianópolis -SC. Brasil.

Aprovado: 23/07/2018

Aprovado: 07/08/2018

Received: 07/23/2018

Approved: 08/07/2018

Recibido: $23 / 07 / 2018$

Aceptado: 07/08/2018

Como citar: Oliveira, C. M., \& Nunes, C. H. S. S. (2018). Modelo de testagem universal aplicado à área da avaliação psicológica. Psicologia: Ciência e Profissão, 38(n.spe), 98-107. https://doi.org/10.1590/1982-3703000209007

How to cite: Oliveira, C. M., \& Nunes, C. H. S. S. (2018). Universal testing model applied to the area of psychological assessment. Psicologia: Ciência e Profissão, 38(n.spe), 98-107. https://doi.org/10.1590/1982-3703000209007

Cómo citar: Oliveira, C. M., \& Nunes, C. H. S. S. (2018). Modelo de prueba universal aplicado al área de evaluación psicológica. Psicologia: Ciência e Profissão, 38(n.spe), 98-107. https://doi.org/10.1590/1982-3703000209007 